\title{
Decision processes in selective attention: Response priming in the Stroop color-word task
}

\author{
W. TRAMMELL NEILL \\ University of South Florida, Tampa, Florida 33620
}

\begin{abstract}
In a random discrete-trials version of the Stroop color-word task, two subsets of trials were compared: (1) trials in which the current distractor word named the color response just made in the preceding trial, and (2) trials in which the current stimulus was unrelated to the previous stimulus. Shorter response latencies and fewer errors in the first condition suggest either that (a) increasing the availability of a response can facilitate its rejection (incompatible with passive strength-of-activation theories), or that (b) the redundancy of the current distractor response and still-potent preceding response reduces the total number of highly activated competing responses present on the current trial. The latter hypothesis is favored by a second experiment in which Stroop stimuli were preceded by a warning signal which "primed" the distractor response, the appropriate color response, an unrelated response, or no response. That the efficiency of selective attention is affected by the number of simultaneous competing responses even when number of input channels remains constant supports theories of attention which place capacity limitations subsequent to stimulus identification.
\end{abstract}

The efficiency of selective attention may be reduced by increasing the number of stimulus input channels to be ignored. Treisman (1964a) found that auditory shadowing is disrupted more by two simultaneous irrelevant messages than by one. Selective listening deteriorates not only with greater physical similarity (voice, apparent location) between relevant and irrelevant inputs, but also with greater discriminability between two competing irrelevant messages. Within the framework of "filter/attenuation" theories of attention (Broadbent, 1958; Treisman, 1964b), irrelevant channels compete for limited perceptual processing capacity, thereby interfering with the identification of the relevant input. Physical similarity effects reflect the complexity of filtering (or attention) required to separate relevant from irrelevant channels.

An opposing point of view is that perceptual processes are automatic and that capacity limitations operate after inputs are identified (Deutsch \& Deutsch, 1963; Keele, 1973; Norman, 1968;

This research was supported by National Institutes of Health General Medical Sciences Training Grant No. 5 T01 GM02165. The experiments were included in a dissertation presented to the University of Oregon, and portions of the data were briefly described at a meeting of the Western Psychological Association in Los Angeles, April 1976. I am grateful to Professors Steven W. Keele, Michael I. Posner, and Douglas L. Hintzman for advice and encouragement. Special thanks are due to Barbara Lewis for computer programming. Requests for reprints should be sent to W. Trammell Neill, Department of Psychology, University of South Florida, Tampa, Florida 33620.
Shiffrin, 1975). From this alternative perspective, varying the number of irrelevant input channels affects the number of active implicit responses which compete for limited output resources. Physical similarity between inputs may then affect the criteria used to discriminate among the competing relevant and irrelevant responses. An implication of this approach to attention is, then, that varying the number of implicitly activated responses while keeping the number of input channels constant should similarly affect the efficiency of selective attention. Such an effect is demonstrated in Experiment 1 by an analysis of sequential relations between trials in a version of the Stroop (1935) color-word task.

\section{EXPERIMENT 1}

In the Stroop task, naming the color of a word is typically slowed when the irrelevant word itself elicits a conflicting color name. Keele (1972) has argued that the Stroop effect itself demonstrates automatic parallel processing of relevant and irrelevant information, resulting in interference only when the irrelevant input activates an incompatible response. Hintzman, Carre, Eskridge, Owens, Shaff, and Sparks (1972) supported Keele's conclusion by showing a relative facilitation of color naming when the distractor word was congruent with the color name. However, interpretation of the Stroop effect is not completely unequivocal. If limited perceptual capacity imposes serial processing of stimulus attributes, a color-related word might "capture" 
attention and thereby delay processing of the color (Hock \& Egeth, 1970). It could be argued that the congruency effect found by Hintzman et al. only shows that benefits of prior response activation outweigh the costs of delayed processing. The issue becomes still more complicated if analysis-by-synthesis approaches are considered, in which the word context might facilitate the actual peceptual processing of the named color (Rosch, 1975) at a cost to processing other colors.

In a random discrete-trials version of the Stroop task, Neill (1977a) compared trials in which the current color matched the distractor word presented in the immediately preceding trial (for example, BLUE written in red preceded by RED written in green) with trials in which the current stimulus was unrelated to the previous stimulus (e.g., BLUE written in red preceded by YELLOW written in green). In one experiment, longer response latencies in the first condition implied selective suppression of competing responses. In the course of pilot work for these experiments, analysis of other obtained relations between trials revealed other interesting phenomena. Of particular interest is the case in which the current distractor word matched the relevant color of the preceding stimulus (e.g., RED in green preceded by BLUE in red)-reverse temporal order from the above suppression test. It was expected that continued availability of the previous color response would strengthen the identical current distractor response, thereby increasing interference relative to the control condition in which the current stimulus was unrelated to the preceding trial. Surprisingly, the opposite result was obtained: The relation between trials produced faster responses than in the unrelated condition. A tentative hypothesis was made that continued availability of the previous color response in the unrelated condition effectively increases the number of activated responses competing on the current trial. For example, if the word RED presented in color green is preceded by the unrelated stimulus YELLOW in color blue, the still-potent response "blue" is added to the current competing response set. In the related condition, the preceding color response is redundant with the current distractor, so the number of current competing responses is not increased. For example, if RED in green is preceded by YELLOW in red, the preceding color red does not activate a response which is not also aciivated by the current stimulus. ${ }^{1}$

\section{Method}

Subjects. Six University of Oregon undergraduates (five male, one female) were paid $\$ 2 / \mathrm{h}$ of participation. Most subjects had previously participated in a variety of orher unrelated reaction time experiments.

Materials and Apparaius. Siroop stimuli consisted of the four words RED, GREEN, BLUE, and YELLOW, writien in each of the four named colors, plus four "control" stimuli of the form
0000 (four zeros) also written in each of the four colors. All stimuli were thinly bordered in black for enhanced contrast and word legibility. Stimuli were rear-projected on an in-line display controlled by a PDP-9 computer, which also recorded reaction time data from four microswitch response keys. At a viewing distance of about $70 \mathrm{~cm}$, the stimuli subtended visual angles roughly from $3.5^{\circ}$ to $7^{\circ}$.

An oscilloscope to the left of the in-line display provided pre. block "ready" information and postblock feedback. Subjects had the option of leaving on or turning off a light which provided dim background illumination.

Procedure. All subjects memorized the following assignment of color names to response keys: "red," left middle finger; "green," left index finger; "blue," right index finger; "yellow," right middle finger. Subjects identified the colors of Stroup stimuli on hand-held cards both verbally and by pointing out the appropriate response key, to verify successful memorization of the kev assignments and ability to discriminate the stimuli.

Each subject was tested individually over 6 days in sessions of approximately $1 \mathrm{~h}$ in length. Each session consisted of 20 blocks of 100 trials each, preceded by one or two practice blocks. On Days $I$ and 6 , the 4 control stimuli and the 4 congruent stimuli (in which the distractor word matched its color name) were interspersed with the 12 conflict stimuli, to verify the existence of the basic Stroop interference effect and its continuation over days of practice. On Days 2-5, 10 blocks of responding to the color of only incongruent (conflict) stimuli were alternated with 10 blocks in which subjects were instructed to respond to the printed word rather than the presented color. These word-relevant blocks were included only to maintain a strong association between the printed word and keypress response, and were not analyzed. Pilot work had suggested that without the interspersed responding to words, the basic interference effect when manually responding to color is greatly attenuated by practice.

On Days 1 and 6 , the subjects received one practice colorrelevant block. On Days $2-5$, they received one color-relevant and one word-relevant practice block, the order varving day to day.

When the word ready appeared on the oscilloscope, the ubject could initiate a block at will by presenting one of the response keys. Each stimulus remained present until the subject's response, at which time it was immediately replaced by a new, randomly determined stimulus, and a new trial was thus begun. After completion of a block of 100 trials, the average reaction time and number correct for that block were displayed on the oscilloscope. The subjects were instructed to emphasize speed of responding, while maintaining $80 \%-90 \%$ accuracy. Subject generally chose to rest up to a minute between blcaks, although longer delays were permitted.

As each Stroop stimulus was randomly chosen, neither the colur nor the distractor word would be predicted from the stimulus. appearing in the preceding trial. The recorded latencies were autoinatically categorized according to whether either the current color or current distractor word matched either the preceding distractor or preceding color. Two subsets of data are of immediate relevance: Approximately 1,000 conflict trials per subject fall into the present "related" category, in which the current distractor matches the preceding response, but not in combination with any other relation (such as between current color and preceding distractor). A comparable number of trials fall into the "unrelated" category, in which neither current color nor distractor are related to the previous stimulus. Other subsets of data irrelevant or ambiguous with regard to present concerns are discussed elsewhere (Neill. 1977a, 1977b).

\section{Results and Discussion}

The mean reaction time for the related condition was $695 \mathrm{msec}$, and for the unrelated condition, $715 \mathrm{msec}$. The 20 -msec difference is highly signif- 
icant, $t(5)=4.75, p<.01$. The error proportions for the related (.12) and unrelated (.17) conditions also differ significantly, $\mathrm{t}(5)=3.32, \mathrm{p}<.05$. Speedaccuracy tradeoff is ruled out by the similar directions of the speed and accuracy effects. ${ }^{2}$

The results are consistent with the hypothesis that continued availability of the previous color response in the unrelated condition increases the number of relatively highly activated responses competing on the current trial. However, before accepting this conclusion, it is necessary to consider possible alternative explanations.

Klein (1964) suggested that allowing subjects to name the current distractor before naming the current color would result in "deactivation" of the distractor response. Klein found that the total time to name the word and then the color of each Stroop stimulus in a list was less than the sum of the times to name the words and colors separately. Unfortunately, the argument is faulty in that it does not allow for any overlap of processing times for the two stimulus attributes. Nevertheless, an analogous explanation might be offered for the present data: Refractoriness of the response just made results in resistance to activation by the current distractor word. However, the "repetition effect" literature indicates that response repetition produces relative facilitation, not response-specific refractoriness, even with minimal response-stimulus interval (RSI) (Rabbitt, 1968), arguing against even a temporary decrease in availability of the particular emitted response. Accordingly, a very strong repetition effect was obtained in the present experiment: When the color response was repeated in the context of a new distractor, mean reaction time was $491 \mathrm{msec}$ (error proportion $=.06)$, and in the same distractor context, $364 \mathrm{msec}(.00)$. Both of these response repetition conditions produced great facilitation relative to the unrelated condition, $t(5)=7.01$ and 11.32 , respectively, both $\mathrm{p}<.001$.

An explanation that cannot be ruled out by these data is that increasing the strength of the distractor response in the related condition actually facilitated the rejection of that response. This hypothesis is incompatible with many theories, especially passive strength-of-activation theories such as Morton's (1969, 1970; Morton \& Chambers, 1973) "logogen" model, in which response tendency is a direct function of strength of activation. However, more complicated hypothesis-testing models, such as Becker's (1976) "verification" model might be extended to account for such a possibility. For example, if hypotheses about the correct response must be tested serially, the Stroop distractor word may first suggest a hypothesis that must be tested and rejected before the correct hypothesis can be tested (Becker, Note 2). If prior activation allows the distractor hypothesis to be made sooner, it will be tested and rejected more quickly, reducing the delay of testing the correct hypothesis.

Experiment 2 was designed to decide between the "number-competition" and "rejection-facilitation" explanations for the relatedness effect found in Experiment 1. The latter predicts that "priming" the distractor word should reduce interference relative to a truly neutral condition in which no response is activated immediately prior to the Stroop stimulus. On the other hand, strength-of-activation theories predict increases in interference when either the distractor or an unrelated response is primed, since interference is viewed as a function of competing response strengths.

\section{EXPERIMENT 2}

\section{Method}

Subjects. Eight new subjects (six male, two female), again mainly undergraduates experienced in other reaction time tasks, were each paid $\$ 2$ for approximately $1 \mathrm{~h}$ of participation.

Materials and Apparatus. The 12 Stroop conflict stimuli from Experiment 1 were again used as test stimuli. The four colored 0000 stimuli from Experiment 1 here served as priming stimuli not to be responded to. In addition, an uncolored plus sign (+) was used as a neutral control for general warning signal effects. The four congruent stimuli were not used.

In all other respects, materials and apparatus were identical to Experiment 1.

Procedure. Each subject was tested individually in one session consisting of 10 blocks, preceded by 1 practice block, of 72 trials each. At the beginning of the session, the subjects memorized the stimulus-response mappings and were pretested as in Experiment 1 . The subjects were then shown the priming and neutral stimuli, and were told that one would be presented as a warning signal before each colored word stimulus to which they had to respond. It was stressed that no aspect of the test stimulus could be predicted from the priming stimulus.

A trial sequence began with the $200-\mathrm{msec}$ presentation of a priming or neutral stimulus, immediately followed by a test conflict stimulus which remained present until the subject's response. The priming and neutral stimuli and the test stimuli were randomly chosen such that each possible pair of priming (or neutral) and test stimuli was sampled without replacement (until all were sampled), except that the frequency of the neutral warning signal was twice that of any one color prime. The resulting proportions of trial types were then 1/3 "neutral" (no color primed), 1/3 "unrelated" (a color other than the distractor and appropriate response color primed), 1/6 "color-related" (correct color response primed), and 1/6 "distractor-related" (distractor response primed)

Following a subject's response, a delay of $500,1,000,2,500$ or $5,000 \mathrm{msec}$ preceded the next trial. It was hoped that the variable delay, randomly chosen, would help to focus the subjects' attention on the priming or neutral stimulus as a warning signal. The relatively long delays between trials probably served to attenuate intertrial effects, which were at any rate balanced by randomization with regard to priming conditions.

Other aspects of procedure were essentially similar to Experiment 1.

\section{Results and Discussion}

The average correct response time for the neutral 
unprimed condition was $725 \mathrm{msec}$ (error proportion $=.08$ ). The color-related priming condition averaged $705 \mathrm{msec}$, the difference from the neutral condition only marginally significant, $t(7)=2.07$, $.05<\mathrm{p}<.10$. However, all but one subject made fewer errors with color-related priming (mean proportion $=.07$ ), resulting in a small, but significant, difference in accuracy, $t(7)=3.55, p<.01$. The errors and latencies in the color-related condition taken together validate the assumption that a response was indeed "primed" prior to the test stimulus, resulting in facilitation when that response was actually appropriate to the test stimulus color.

Both the unrelated condition $(764 \mathrm{msec}$ ) and the distractor-related condition $(771 \mathrm{msec})$ were significantly slower than the neutral condition, $t(7)=3.42$ and 3.34 , respectively, both $p<.02$. The error proportions, .07 and .08 , respectively, were not significantly different from the neutral condition. None of the differences between the unrelated and distractorrelated conditions are significant, although six out of eight subjects tended to be slower in the distractorrelated condition, and six made more errors in that condition also.

The increased interference in the distractor-related priming condition casts doubt on the proposition that increasing the availability of a response can facilitate its rejection. Since both the distractorrelated and unrelated conditions produced increases in reaction time, it seems reasonable to conclude that there are two possible sources of interference in the Stroop conflict: (1) the number of relatively highly activated responses competing simultaneously, and (2) the strength of activation of each competing response.

\section{GENERAL DISCUSSION}

The results of Experiments 1 and 2 taken together demonstrate that the efficiency of selective attention is affected by both the number of competing implicit responses and the strength of each individual competing implicit response. This conclusion is easily viewed in the context of the "logogen" model proposed by Morton (1970). A logogen is an abstract memory representation associated with a verbal labeling response. Any stimulus input relevant to a logogen increases the strength of that logogen by adding to its count of sensory features. When the feature count exceeds a criterion, that response becomes eligible to the limited-capacity response buffer. However, the strength of a given response must be evaluated against a fluctuating noise background produced by other logogens. According to the principles of signal detection theory, the decision processes involved in allocating output resources would then be affected by any inputs adding to the feature counts of irrelevant logogens.
In Experiment 2, priming an unrelated response and priming the distractor response produced comparable increases in reaction time. In contrast, priming an unrelated response in Experiment 1 was more potent-otherwise no relatedness effect could have been obtained. While the two patterns are not identical, they are both consistent with a general view of interference as a function of both number of competing responses and the relative strength (or availability) of each competing response. In the absence of a more precise statistical decision model, it is difficult to specify when one source of interference should contribute more than the other. The important point is that number of competing responses influences interference in Experiment 1, where number of input channels remains constant, the major alternative explanation having been ruled out by Experiment 2.

As discussed in the introduction, an effect of number of competing responses, even when number of input channels is held constant, is a necessary prediction of theories that hold that selective attention is mostly a matter of response selection. The effect of number here must be on the decision processes that have to choose between two or more competing responses. However, the results do not wholly discredit the opposing view that perceptual selection is important. Indeed, more recent statements of filter/attenuation theory (Broadbent, 1971; Treisman, 1969) concede that response competition may occur if perceptual capacity is not overloaded and the stimuli do not provide sufficient criteria for selective filtering or attenuation. Nevertheless, the present results must lend support to those (e.g., Deutsch \& Deutsch, 1963; Keele, 1973; Norman, 1968; Shiffrin, 1975) who claim that postcategorical capacity limitations are sufficient to account for attentional phenomena, including the effects of irrelevant input channels.

\section{REFERENCE NOTES}

1. Keele, S. W. Unpublished data, 1975.

2. Becker, C. A. The recognition of words. Unpublished manuscript.

\section{REFERENCES}

BECKER, C. A. Allocation of attention during visual word recognition. Journal of Experimental Psychology: Human Perception and Performance, 1976, 2, 556-567.

Broadbent, D. E. Perception and communication. London: Pergamon, 1958.

Broadbent, D. E. Decision and stress. London: Academic Press, 1971.

Deutsch. J. A., \& Deutsch, D. Attention: Some theoretical considerations. Psychological Review, 1963, 70, 80-90.

Hintzman, D. L., Carre, F. A., Eskridge, V. L., Owens, A. M. Shaff, S. S., \& Sparks, M. E. "Stroop" effect: Input or output phenomenon? Journal of Experimental Psychology, 1972, 95, 458-459.

Hock, H. S., \& EGETH, H. Verbal interference with encoding 
in a perceptual classification task. Journal of Experimental Psychology, 1970, 83, 299-303.

KeELE, S. W. Attention demands of memory retrieval. Journal of Experimental Psychology, 1972, 93, 245-248.

KeEle, S. W. Attention and human performance. Pacific Palisades, Calif: Goodyear, 1973.

KLEIN, G. S. Semantic power measured through the interference of words with color naming. American Joumal of Psychology, 1964, 77, 576-588.

Morton, J. Categories of interference: Verbal mediation and conflict in card sorting. British Journal of Psychology, 1969, 60, 329-346.

Morton, I. A functional model for memory. In D. A. Norman (Ed.), Models of human memory. New York: Academic Press, 1970.

Morton, J., \& Chambers, S. M. Selective attention to words and colours. Quarterly Joumal of Experimental Psychology, 1973, 25, 387-397.

NeILL, W. T. Inhibitory and facilitatory processes in selective attention. Joumal of Experimental Psychology: Human Perception and Performance, 1977, 3, 444-450. (a)

NeILL, W. T. Attention: The coordination of internal codes. Unpublished dissertation, University of Oregon, 1977. (b)

Norman, D. A. Toward a theory of memory and attention. Psychological Review, 1968, 75, 522-536.

RabitT, P. M. A. Repetition effects and signal classification strategies in serial choice-response tasks. Quarterly Journal of Experimental Psychology, 1968, 20, 232-240.

Rosch, E. The nature of mental codes for color categories. Journal of Experimental Psychology: Human Perception and Performance, 1975, 1, 303-322.

SHIFFRIN, R. M. The locus and role of attention in memory systems. In P. M. A. Rabbitt \& S. Dornic (Eds.), Attention and performance V. New York: Academic Press, 1975.

Stroop, J. R. Studies of interference in serial verbal reactions. Journal of Experimental Psychology, 1935, 18, 643-662.

Treisman, A. M. The effect of irrelevant material on the efficiency of selective listening. American Journal of Psychology, 1964, 77, 533-546. (a)

Treisman, A. M. Monitoring and storage of irrelevant messages in selective attention. Journal of Verbal Learning and Verbal Behavior, 1964, 3, 449-459. (b)

Treisman, A. M. Strategies and models of selective attention. Psychological Review, 1969, 76, 282-299.

\section{NOTES}

1. Depending on experimental context, the preceding distractor may also remain available (Neill, 1977a). If so, it should contribute equally to the present related and unrelated conditions. Contributions from trials twice or more removed from the current trial are balanced by randomization. In this context, "response activation" must be viewed not as absolute, but relative to a background of weaker, less recently activated responses.

2. A subsequent analysis of comparable Stroop task data collected by Keele (Note 1) reveals a similar paltern over a larger subject sample $(n=32)$, without interspersed word-relevant blocks and a 50-msec RSI.

(Received for publication September 27, 1977; accepted for publication November 9, 1977.) 